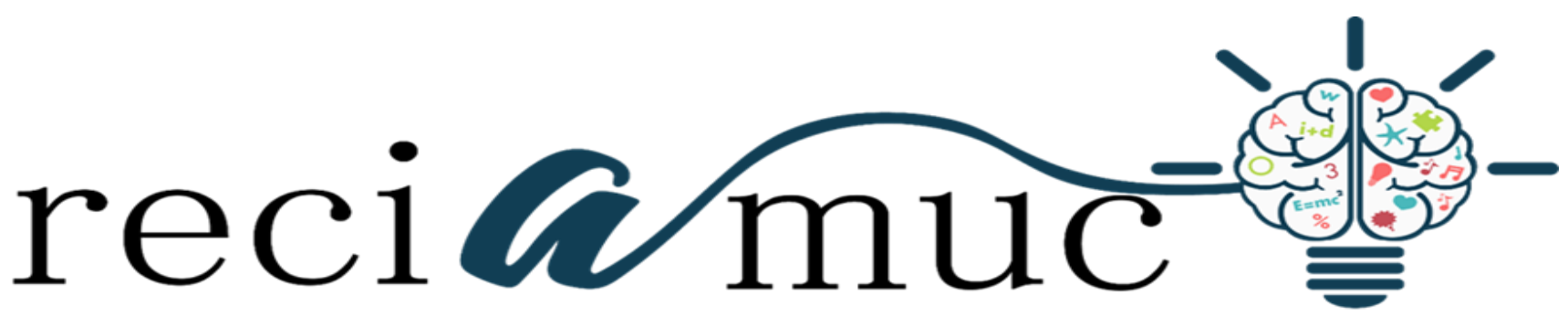

Revista cientifica de investigación actualización del mundo de las ciencias

Paola Katherine Muñoz Cedeño ${ }^{\text {a }}$ J Jorge Efraín Camacho Desiderio ${ }^{\text {b; }}$ Ornella Lorena Alvarado Chiquito ${ }^{c}$; Miguel Melquiades Macías Mora ${ }^{\mathrm{d}}$

La dismenorrea como principal causante de periodos menstruales doloroso

Dysmenorrhea as the main cause of painful menstrual periods

Revista Científica de Investigación actualización del mundo de las Ciencias. Vol. 3 núm., 3, julio, ISSN: 2588-0748, 2018, pp. 769-788

DOI: $10.26820 /$ reciamuc/3.(3).julio.2019.769-788

URL: $\underline{\text { http://reciamuc.com/index.php/RECIAMUC/article/view/302 }}$

Código UNESCO: 3205 Medicina Interna

Tipo de Investigación: Artículo de Revisión

(C) RECIAMUC; Editorial Saberes del Conocimiento, 2019

Recibido: 28/04/2019

Aceptado: 19/05/2019

Publicado: 01/07/2019

Correspondencia: katherin_4689@hotmail.com
a. Médico; Investigador Independiente; Guayaquil, Ecuador; katherin_4689@ hotmail.com
b. Médico; Investigador Independiente; Guayaquil, Ecuador.
c. Médico; Investigador Independiente; Guayaquil, Ecuador; loreal1101@ hotmail.com
d. Médico; Investigador Independiente; Guayaquil, Ecuador. 


\section{La dismenorrea como principal causante de periodos menstruales doloroso}

Vol. 3, núm. 3., (2019)

Paola Katherine Muñoz Cedeño; Jorge Efraín Camacho Desiderio; Ornella Lorena Alvarado Chiquito; Miguel Melquiades Macías Mora

\section{RESUMEN}

La dismenorrea es una de las causas más comunes de dolor pélvico. Afecta negativamente la calidad de vida de los pacientes y, en ocasiones, resulta en una restricción de la actividad. Un historial y un examen físico, incluido un examen pélvico en pacientes que han tenido relaciones sexuales vaginales, puede revelar la causa. La dismenorrea primaria es el dolor menstrual en ausencia de patologías pélvicas. Hemorragia uterina anormal, dispareunia, dolor no cíclico, cambios en la intensidad y duración del dolor, y los hallazgos anormales del examen pélvico sugieren una patología subyacente (dismenorrea secundaria) y requieren más investigación. Se debe realizar una ecografía transvaginal si se sospecha una dismenorrea secundaria. La endometriosis es la causa más común de dismenorrea secundaria. Los síntomas y signos de adenomiosis incluyen dismenorrea, menorragia, y un útero uniformemente agrandado. Las opciones de manejo para la dismenorrea primaria incluyen medicamentos antiinflamatorios no esteroideos y anticonceptivos hormonales. Los anticonceptivos hormonales son el tratamiento de primera línea para la dismenorrea causada por la endometriosis. El calor tópico, el ejercicio y la suplementación nutricional pueden ser beneficiosos para pacientes con dismenorrea; sin embargo, no hay pruebas suficientes para apoyar el uso del yoga, la acupuntura o masaje.

Palabras Claves: Dismenorrea; Dolor Pélvico; Diagnóstico; Tratamiento. 


\title{
La dismenorrea como principal causante de periodos menstruales doloroso
}

Vol. 3, núm. 3., (2019)

Paola Katherine Muñoz Cedeño; Jorge Efraín Camacho Desiderio; Ornella Lorena Alvarado

Chiquito; Miguel Melquiades Macías Mora

\begin{abstract}
Dysmenorrhea is one of the most common causes of pelvic pain. It negatively affects the quality of life of patients and, sometimes, results in a restriction of activity. A history and a physical exam, including a pelvic exam in patients who have had vaginal sex, can reveal the cause. Primary dysmenorrhea is menstrual pain in the absence of pelvic pathologies. Abnormal uterine bleeding, dyspareunia, noncyclic pain, changes in the intensity and duration of pain, and abnormal findings of pelvic examination suggest an underlying pathology (secondary dysmenorrhea) and require further investigation. A transvaginal ultrasound should be performed if secondary dysmenorrhea is suspected. Endometriosis is the most common cause of secondary dysmenorrhea. Symptoms and signs of adenomyosis include dysmenorrhea, menorrhagia, and a uniformly enlarged uterus. Management options for primary dysmenorrhea include non-steroidal anti-inflammatory drugs and hormonal contraceptives. Hormonal contraceptives are the first-line treatment for dysmenorrhea caused by endometriosis. Topical heat, exercise and nutritional supplementation may be beneficial for patients with dysmenorrhea; however, there is not enough evidence to support the use of yoga, acupuncture or massage.
\end{abstract}

Key Words: Dysmenorrhea; Pelvic Pain; Diagnosis; Treatment. 


\section{La dismenorrea como principal causante de periodos menstruales doloroso}

Vol. 3, núm. 3., (2019)

Paola Katherine Muñoz Cedeño; Jorge Efraín Camacho Desiderio; Ornella Lorena Alvarado Chiquito; Miguel Melquiades Macías Mora

\section{Introducción.}

La dismenorrea es un problema ginecológico muy frecuente. Como el dolor es muy subjetivo y como tal, difícil de evaluar, los estudios muestran un amplio rango de prevalencia 1781\% (P, Latthe, \& Say, 2006). La percepción del dolor también está influenciada por los antecedentes culturales. Esto explica que diferentes prevalencias se encuentren en diferentes partes del mundo. La mayoría de los estudios se ocupan de la dismenorrea en entornos industrializados y hay datos de disponibilidad del poder sin los datos de entornos con recursos limitados. Esto no significa, sin embargo, que la dismenorrea no sea tan importante en esos entornos. Como las causas de dismenorrea son las mismas en todo el mundo se puede asumir que como muchas mujeres se ven afectadas por ella en entornos de escasos recursos como en las regiones industrializadas. Los estudios mencionados anteriormente encontraron que el $15 \%$ de los participantes mencionó el dolor a ser grave e interfiriendo con sus actividades normales. En un estudio, el $35 \%$ de los estudiantes secundarios femeninos informó faltar a la escuela (Banikarim, Chacko, \& Kelder, 2000). Si se tiene en cuenta que las actividades diarias en entornos de bajos recursos son físicamente mucho más difícil que en los países industrializados, y que las cir-cunstancias para las niñas que van a la escuela son mucho más difíciles en muchas partes del mundo, se puede imaginar la efectividad del tratamiento para este condicion común puede influir significativamente en el rendimiento socio-económico y el bienestar social.

Además, la dismenorrea tiene un aspecto psicológico, como las mujeres y especialmente las adolescentes a menudo sufren la preocupación de que algo podría estar mal con sus órganos reproductivos, lo que dificulta su fertilidad en los últimos años. Esto puede causar mucho miedo y también debe ser abordado. En las adolescentes dismenorrea puede ser el primer contacto con 


\section{La dismenorrea como principal causante de periodos menstruales doloroso}

Vol. 3, núm. 3., (2019)

Paola Katherine Muñoz Cedeño; Jorge Efraín Camacho Desiderio; Ornella Lorena Alvarado Chiquito; Miguel Melquiades Macías Mora

un servicio de salud reproductiva. Esta puede ser una oportunidad para el asesoramiento sobre otras cuestiones tales como las infecciones de transmisión sexual (ITS) y la anticoncepción y ayudar a la niña para establecer un vínculo de por vida a los servicios de salud reproductiva. Una condición previa para esto es que la primera visita funcione bien para la chica y no es, como a menudo, traumatizante.

\section{Metodología.}

Para el desarrollo de este proceso investigativo, se plantea como metodología la encaminada hacia una orientación científica particular que se encuentra determinada por la necesidad de indagar en forma precisa y coherente una situación, en tal sentido (Davila, 2015) define la metodología "como aquellos pasos previos que son seleccionados por el investigador para lograr resultados favorables que le ayuden a plantear nuevas ideas”.(p.66)

Lo citado por el autor, lleva a entender que el desarrollo de la acción investigativa busca simplemente coordinar acciones enmarcadas en una revisión bibliográfica con el fin de complementar ideas previas relacionadas a la dismenorrea y su incidencia en el dolor pélvico menstrual a través de una revisión de literatura, para así finalmente elaborar un cuerpo de consideraciones generales que ayuden a ampliar el interés propuesto.

\section{Tipo de Investigación}

Dentro de toda práctica investigativa, se precisan acciones de carácter metodológico mediante las cuales, se logra conocer y proyectar los eventos posibles que la determinan, así como las características que hacen del acto científico un proceso interactivo ajustado a una 


\section{La dismenorrea como principal causante de periodos menstruales doloroso}

Vol. 3, núm. 3., (2019)

Paola Katherine Muñoz Cedeño; Jorge Efraín Camacho Desiderio; Ornella Lorena Alvarado Chiquito; Miguel Melquiades Macías Mora

realidad posible de ser interpretada. En este sentido, se puede decir, que la presente investigación corresponde al tipo documental, definido por Castro (2016), "se ocupa del estudio de problemas planteados a nivel teórico, la información requerida para abordarlos se encuentra básicamente en materiales impresos, audiovisuales y /o electrónicos”. (p.41).

En consideración a esta definición, la orientación metodológica permitió la oportunidad de cumplir con una serie de actividades inherentes a la revisión y lectura de diversos documentos donde se encontraron ideas explicitas relacionadas con los tópicos encargados de identificar a cada característica insertada en el estudio. Por lo tanto, se realizaron continuas interpretaciones con el claro propósito de revisar aquellas apreciaciones o investigaciones propuestas por diferentes investigadores relacionadas con el tema de interés, para luego dar la respectiva argumentación a los planteamientos, en función a las necesidades encontradas en la indagación.

\section{Fuentes Documentales}

El análisis correspondiente a las características que predomina en el tema seleccionado, llevan a incluir diferentes fuentes documentales encargadas de darle el respectivo apoyo y en ese sentido cumplir con la valoración de los hechos a fin de generar nuevos criterios que sirven de referencia a otros procesos investigativos. Para (CASTRO, 2016) las fuentes documentales incorporadas en la investigación documental o bibliográfica, "representa la suma de materiales sistemáticos que son revisados en forma rigurosa y profunda para llegar a un análisis del fenómeno".(p.41). Por lo tanto, se procedió a cumplir con la realización de una lectura previa determinada para encontrar aquellos aspectos estrechamente vinculados con el tema, con el fin de explicar mediante un desarrollo las respectivas apreciaciones generales de importancia. 


\section{La dismenorrea como principal causante de periodos menstruales doloroso}

Vol. 3, núm. 3., (2019)

Paola Katherine Muñoz Cedeño; Jorge Efraín Camacho Desiderio; Ornella Lorena Alvarado

Chiquito; Miguel Melquiades Macías Mora

\section{Técnicas para la Recolección de la Información}

La conducción de la investigación para ser realizada en función a las particularidades que determinan a los estudios documentales, tiene como fin el desarrollo de un conjunto de acciones encargadas de llevar a la selección de técnicas estrechamente vinculadas con las características del estudio. En tal sentido, (Bolívar, 2015), refiere, que es "una técnica particular para aportar ayuda a los procedimientos de selección de las ideas primarias y secundarias”. (p. 71).

Por ello, se procedió a la utilización del subrayado, resúmenes, fichaje, como parte básica para la revisión y selección de los documentos que presentan el contenido teórico. Es decir, que mediante la aplicación de estas técnicas se pudo llegar a recoger informaciones en cuanto a la revisión bibliográfica de los diversos elementos encargados de orientar el proceso de investigación. Tal como lo expresa, (Bolívar, 2015) "las técnicas documentales proporcionan las herramientas esenciales y determinantes para responder a los objetivos formulados y llegar a resultados efectivos" (p. 58). Es decir, para responder con eficiencia a las necesidades investigativas, se introdujeron como técnica de recolección el método inductivo, que hizo posible llevar a cabo una valoración de los hechos de forma particular para llegar a la explicación desde una visión general.

Asimismo, se emplearon las técnicas de análisis de información para la realización de la investigación que fue ejecutada bajo la dinámica de aplicar diversos elementos encargados de determinar el camino a recorrer por el estudio, según, (Bolívar, 2015) las técnicas de procesamiento de datos en los estudios documentales "son las encargadas de ofrecer al investigador la visión o pasos que debe cumplir durante su ejercicio, cada una de ellas debe estar 


\section{La dismenorrea como principal causante de periodos menstruales doloroso}

Vol. 3, núm. 3., (2019)

Paola Katherine Muñoz Cedeño; Jorge Efraín Camacho Desiderio; Ornella Lorena Alvarado Chiquito; Miguel Melquiades Macías Mora

en correspondencia con el nivel a emplear" (p. 123). Esto indica, que para llevar a cabo el procesamiento de los datos obtenidos una vez aplicado las técnicas seleccionadas, tales como: fichas de resumen, textual, registros descriptivos entre otros, los mismos se deben ajustar al nivel que ha sido seleccionado.

\section{Resultados.}

La dismenorrea se describe como el dolor cíclico o recurrente de los períodos menstruales. El dolor suele ser similar a calambres, cólicos, situada en la región suprapúbica con radiación a la zona baja de la espalda y las piernas y se mantiene por lo general 24-48 h. A menudo, las mujeres describen acompañamiento de síntomas tales como diarrea, náuseas, hinchazón y cansancio. Hay dos formas de la dismenorrea. Esta diferenciación es importante como se verá.

La dismenorrea primaria comienza por definición alrededor de la menarquia y describe el dolor durante el período menstrual sin ninguna causa subyacente. Así que la mayoría de los pacientes que se verán para la dismenorrea primaria será de menor edad. La aparición del dolor suele ser de unas pocas horas antes de que comience el flujo sanguíneo y tendrá una duración de uno a dos días del período. A menudo, la dismenorrea primaria se vuelve menos con la edad o después del parto.

La dismenorrea secundaria se refiere a dolor durante períodos menstruales con una patología subyacente. Como cuestión de hecho, los síntomas sólo se iniciarán después que la causa subyacente se ha desarrollado. Los pacientes con dismenorrea secundaria serán en su 


\section{La dismenorrea como principal causante de periodos menstruales doloroso}

Vol. 3, núm. 3., (2019)

Paola Katherine Muñoz Cedeño; Jorge Efraín Camacho Desiderio; Ornella Lorena Alvarado Chiquito; Miguel Melquiades Macías Mora

mayoría mujeres más maduras. A menudo, el dolor se inicia muchas horas o incluso días antes del inicio del flujo sanguíneo y puede durar todo el período.

\section{Causas de la dismenorrea}

\section{La dismenorrea primaria}

Esto es causado por una producción excesiva de prostaglandinas en el cuerpo justo antes de que comienza la menstruación. Esto es debido a la caída de la progesterona que induce el desprendimiento del revestimiento endometrial para prepararse para la menstruación y, a través de este, el aumento de la producción de prostaglandina. La prostaglandina es una hormona, que se puede saber a partir de la obstetricia como el misoprostol. Los síntomas adicionales de dismenorrea como náuseas y diarrea son los mismos que los efectos secundarios de misoprostol.

La prostaglandina es una especie de subproducto de cambios en la menstruación de endometrio y no se sabe por qué algunas mujeres producen más que otras, pero esto parece ser sólo posible cuando los ciclos menstruales son ovulatorios (con una ovulación ) (Leyland, Casper, Laberge, \& Singh, 2010). El hecho de que a menudo los primeros ciclos en una chica son anovulatorios explica por qué la dismenorrea primaria comienza a menudo sólo unos pocos ciclos después de la menarquia. El exceso de prostaglandinas conduce a la hiperfrecuencia, las contracciones uterinas disrítmicas y reducción de flujo sanguíneo uterino, lo que conduce al dolor. Muy rara vez, la dismenorrea primaria es causada por malformaciones congénitas o adquiridas del tracto reproductor femenino, tales como el himen imperforado, y la obstrucción después de la mutilación genital femenina. 


\section{La dismenorrea como principal causante de periodos menstruales doloroso}

Vol. 3, núm. 3., (2019)

Paola Katherine Muñoz Cedeño; Jorge Efraín Camacho Desiderio; Ornella Lorena Alvarado Chiquito; Miguel Melquiades Macías Mora

Es bueno saber que la dismenorrea primaria puede ser muy doloroso, pero que siempre es inofensivo y no interfiere con la función reproductiva.

\section{La dismenorrea secundaria}

Las posibles causas de la dismenorrea secundaria son:

- Endometriosis (células endometriales que crecen fuera del lado del útero, que conducen a los quistes y adherencias).

- Adenomiosis (células endometriales que crecen dentro de la pared uterina en la capa muscular llamada miometrio).

- $\quad$ Los fibromas uterinos (tumores benignos de la pared uterina).

- $\quad$ ITS Aguda y enfermedad inflamatoria pélvica crónica.

- Las adherencias pélvicas (postoperatorio o postinfecciosa).

- Cánceres del aparato reproductor (signo tardío).

En la mayoría de estas condiciones, el dolor menstrual está relacionado a la presión del tejido deteriorado (fibromas, adenomiosis, cáncer) y expulsión de coágulos de sangre de la cavidad uterina que conduce a contracciones disrítmicas. En algunos, de tracción (adherencias, PID), o la producción de prostaglandinas o factores de tipo de las prostaglandinas (ITS aguda, endometriosis) podrían desempeñar un papel (French, 2005). 


\section{La dismenorrea como principal causante de periodos menstruales doloroso}

Vol. 3, núm. 3., (2019)

Paola Katherine Muñoz Cedeño; Jorge Efraín Camacho Desiderio; Ornella Lorena Alvarado

Chiquito; Miguel Melquiades Macías Mora

\section{Tomar la historia}

- Quejas reales: tipo de dolor, la radiación, la aparición en relación con el ciclo menstrual y periodo. Otros síntomas: Cuidado con los síntomas que acompañan lo antes mencionados, tales como diarrea y náuseas. La endometriosis puede crecer en la vejiga y el intestino y causar hematuria y sangre en las heces. Preguntar acerca de dolor durante el coito (dis-pareunia) haciendo alusión a la endometriosis, adenomiosis, fibromas o PID. También preguntar por disquecia (dolor durante la defecación), que es una señal para la endometriosis intestinal.

- Duración de las quejas (Primaria / secundaria, el número de meses / años). Cuidado con: cada nueva aparición dismenorrea puede ser un embarazo ectópico equivocado

- Duración de los períodos y la regularidad del ciclo: Adenomiosis y fibromas suelen ir junto con la menorragia (mayor pérdida de sangre y la menstruación prolongada). Manchado y sangrado irregular pueden ser un signo de ITS. Sangrado después de la relación sexual o durante puede hacer alusión a las ITS, sino también en el cáncer de cuello uterino.

- Paridad, operaciones pélvicas: Subfertilidad puede ser una señal para la endometriosis, fibromas y PID; aborto recurrente puede hacer alusión a la existencia de los fibromas uterinos. Operaciones pélvicas pueden causar adherencias o dar lugar a infecciones que a su vez pueden causar PID. Legrados 


\section{La dismenorrea como principal causante de periodos menstruales doloroso}

Vol. 3, núm. 3., (2019)

Paola Katherine Muñoz Cedeño; Jorge Efraín Camacho Desiderio; Ornella Lorena Alvarado Chiquito; Miguel Melquiades Macías Mora

llevados a cabo bajo condiciones estériles son un factor de riesgo muy importante en este contexto, pero a menudo se pierden durante la historia clínica.

- $\quad$ El uso de anticonceptivos puede aliviar el dolor en las dismenorreas primarias y la endometriosis.

- Deseo de embarazo con el fin de determinar el uso de anticonceptivos orales para la terapia.

- Fiebre, descarga y dolor abdominal puede ser un signo de infecciones de transmisión sexual, pero también puede ser un signo tardío de cáncer de cuello uterino.

- El estrés o una historia familiar de la dismenorrea: Dismenorrea primaria puede ser psicosomático. La manera en que parientes mujeres de más edad se ocupan de su período menstrual puede influir en la percepción de las niñas acerca de la condición de mujer y la menstruación y la forma en que tratan los períodos menstruales dolorosos. El estrés en la escuela o en torno a temas de la adolescencia y crecer puede conducir a un cambio en la percepción del dolor y la dismenorrea primaria también.

Por favor tener en cuenta que un período menstrual es el signo inevitable que un embarazo no funcionó. Por lo tanto, para las mujeres con dificultades para concebir o en los casos en los que su familia o la sociedad en la ley ejercen presión sobre ellos para concebir, cada periodo es 'doloroso' de una manera psicológica. 


\section{La dismenorrea como principal causante de periodos menstruales doloroso}

Vol. 3, núm. 3., (2019)

Paola Katherine Muñoz Cedeño; Jorge Efraín Camacho Desiderio; Ornella Lorena Alvarado Chiquito; Miguel Melquiades Macías Mora

\section{Examen físico}

La mayoría de las veces se puede hacer el diagnóstico de salida con muchas investigaciones en sus instalaciones. La dismenorrea primaria es un diagnóstico de exclusión, pero su historial con el inicio de los síntomas y la menarquia ya se le puede ayudar a establecer el diagnóstico. En la dismenorrea primaria su examen fuera de la menstruación será normal ya que no hay causa subyacente. La mayoría de las mujeres acuden al médico cuando tienen dolor, por lo que sería prudente para volver a examinar una vez su período ha terminado de ver la diferencia. Estar al tanto de posibles malformaciones congénitas como un cuerno uterino rudimentario que puede causar el dolor.

Tenga en cuenta: una mujer puede comenzar con la dismenorrea primaria y con la experiencia de tiempo de los síntomas secundarios adicionales debido a las nuevas causas subyacentes. Así que preguntar a cada mujer si el dolor ha cambiado con el tiempo, especialmente en relación con inicio y duración. Se debe hacer un examen ginecológico completo en todos los pacientes con dismenorrea para establecer o descartar causas subyacentes.

\section{La palpación abdominal}

Al hacer un examen abdominal se puede evaluar masas abdominales (fibromas, cáncer) o puntos de resistencia relacionada con el dolor (PID, adherencias) (Morrow \& Naumburg, 2009). Se debe buscar cicatrices y evaluar su curación. Una amplia y fea cicatriz podría ser una señal de sanación secundaria con la probabilidad de infección o adherencias.

\section{Examen con espéculo}




\section{La dismenorrea como principal causante de periodos menstruales doloroso}

Vol. 3, núm. 3., (2019)

Paola Katherine Muñoz Cedeño; Jorge Efraín Camacho Desiderio; Ornella Lorena Alvarado Chiquito; Miguel Melquiades Macías Mora

Durante un examen con espéculo se pueden encontrar signos de cáncer de cuello uterino (hemorragia, úlceras, erosiones, masas) o para ITS (flujo vaginal anormal, una superficie del cuello uterino rojiza, la descarga del orificio cervical) y para la endometriosis vaginal (ampollas de color rojo oscuro o púrpura o manchas). El cuello uterino puede ser distorsionada a un lado por los fibromas, masas tuboováricos o endometriosis, o se acorta por el crecimiento del fibroma cervical o intracavitaria.

\section{El examen vaginal}

Durante la palpación bimanual se puede encontrar signos de adenomiosis (agrandado, suave, útero menudo tierna) o fibromas (agrandado, útero firme, el útero gran volumen, con jorobas, móviles o inmóviles) y las masas ováricas tales como tumores ováricos benignos o malignos y abscesos tubo-ovárico como un signo de PID (masas derecha, izquierda o detrás del útero, móvil o im-móvil). ternura cervical puede ser un signo de infección aguda o crónica (STI, PID), sino también para endometriosis y embarazo ectópico. A la palpación dolorosa del fornix posterior puede ser una muestra de endometriosis del espacio Douglas' o infiltración del tejido entre la vagina y el intestino.

\section{Examen rectal}

Un examen rectal puede revelar sangre o dolor anal que pueden ser signos de endometriosis y, a veces la endometriosis en tabique recto-vaginal puede ser palpado por examen recto-vaginal. 


\section{La dismenorrea como principal causante de periodos menstruales doloroso}

Vol. 3, núm. 3., (2019)

Paola Katherine Muñoz Cedeño; Jorge Efraín Camacho Desiderio; Ornella Lorena Alvarado Chiquito; Miguel Melquiades Macías Mora

\section{Investigaciones más profundas}

Ecografía vaginal / abdominal

Si el diagnóstico clínico es dismenorrea secundaria se puede echar un vistazo a las siguientes patologías:

Fibras uterinas Su ecogenicidad en la ecografía es un poco menos de miometrio normal. Por lo general tienen bordes bien definidos en el miometrio y una cápsula. No se debe olvidar de hacer una ecografía abdominal, así como a veces se perderá en la ecografía vaginal si son grandes y fuera de la pelvis.

Adenomiosis La adenomiosis es difícil ver con ultrasonido y el diagnóstico es a menudo uno de exclusión. La pared posterior del útero podría ser más gruesa que la pared anterior y es posible encontrar áreas de hiperrefringencia en ella. Los pacientes con adenomiosis a menudo sufren de trastornos menstruales, junto con la dismenorrea.

Endometrioma Endometrioma a menudo aparecen como tumores en los ovarios. Si son lo suficientemente grande como es posible que se vean en la ecografía. Por lo general son quística con una disminución o ausente ecogenicidad y pueden parecerse a los quistes ováricos o hidrosalpinx.

Absceso tubo-ovárico, hidrosalpinx, absceso pélvico Las infecciones pélvicas pueden provocar abscesos en varios lados. 


\section{La dismenorrea como principal causante de periodos menstruales doloroso}

Vol. 3, núm. 3., (2019)

Paola Katherine Muñoz Cedeño; Jorge Efraín Camacho Desiderio; Ornella Lorena Alvarado Chiquito; Miguel Melquiades Macías Mora

Prueba de embarazo en orina / análisis de orina

Cada paciente con nuevo dolor pélvico debe tener una prueba de embarazo en orina para excluir un embarazo ectópico, incluso si ella dice que ella ha tenido su período. Hacer un análisis de orina para descartar la infección crónica del tracto urinario y la esquistosomiasis en las zonas endémicas.

Velocidad de sedimentación globular / recuento de glóbulos blancos

Estos pueden ayudar a confirmar o excluir una causa infecciosa del dolor pélvico tales como masas tuboováricos o PID.

\section{Tratamiento}

La terapia para la dismenorrea primaria es muy simple y barato:

- $\quad$ Para las mujeres con el deseo de anticoncepción de una píldora anticonceptiva normal (OCP) con <35 mg estrógeno y progesterona ha mostrado algún efecto en muchos ensayos, aunque la evidencia no es firme, debido a los diseños de los estudios (Wong, Farquhar, Roberts, \& Proctor, 2009). También se puede rastrear el OCP no cíclico: la mujer no obtendrá un período y no tendrá la dismenorrea.

- Para todos los otros pacientes con dismenorrea primaria cualquier antinflamatorio no esteroideo (AINE: aspirina, diclofenaco, ibuprofeno, indometacina) es muy eficaz. No hay ningún estudio que demuestra la superioridad de una de esos fármacos anteriores AINE, pero todos son más eficaces que el placebo, 


\section{La dismenorrea como principal causante de periodos menstruales doloroso}

Vol. 3, núm. 3., (2019)

Paola Katherine Muñoz Cedeño; Jorge Efraín Camacho Desiderio; Ornella Lorena Alvarado Chiquito; Miguel Melquiades Macías Mora

paracetamol o butilescopolamina (Buscopan $\left.{ }^{\circledR}\right)$. Esto es debido a su modo de acción: Los AINEs detienen la producción de Prostaglandina, algo que no pueden hacer el paracetamol o BUSCAPINA.

- Los AINE sólo reducen la producción de Prostaglandinas. No reducen el efecto de pros-taglandina en el útero. Una vez que las prostaglandinas están en la circulación que causa dolor, los AINE no funcionarán con la mayor eficacia.

Por lo general, las mujeres con dismenorrea primaria saben cuándo el dolor se inicia y el tiempo que normalmente se queda y hay sólo unos pocos ciclos en los que no se experimenta dolor. Por lo tanto, para un tratamiento exitoso y un buen cumplimiento, hay tres mensajes importantes que se debe dar a su paciente:

- $\quad$ Siempre tener sus AINE al alcance cuando se encuentre cerca de su período.

- $\quad$ Siempre tomar sus AINE tan pronto como se sienta el dolor que viene. No espere hasta que el dolor es muy fuerte ya que esto es demasiado tarde.

- $\quad$ Siempre tome sus AINE siempre y cuando el dolor por lo general se mantiene.

Es importante prescribir medicamentos durante 3 meses y luego ver de nuevo al paciente para evaluar junto con ella si vio algo de alivio. Si no es así, cambiar a otro AINE o añadir un anticonceptivo oral si no se planea el embarazo. 


\section{La dismenorrea como principal causante de periodos menstruales doloroso}

Vol. 3, núm. 3., (2019)

Paola Katherine Muñoz Cedeño; Jorge Efraín Camacho Desiderio; Ornella Lorena Alvarado Chiquito; Miguel Melquiades Macías Mora

\section{Fármacos no esteroideos antiinflamatorios para la dismenorrea}

- Indometacina comprimidos de $25 \mathrm{mg}$ tres veces durante 48-72 $\mathrm{h}$ cada menstruación.

- $\quad$ El ibuprofeno comprimido de $400 \mathrm{mg}$ tres veces durante 48-72 $\mathrm{h}$ cada menstruación.

- diclofenacos comprimidos de $50 \mathrm{mg}$ tres veces durante 48-72 $\mathrm{h}$ cada menstruación.

El tratamiento de la dismenorrea secundaria difiere de acuerdo con las causas subyacentes. El dolor va a detener o disminuir una vez que la causa subyacente es tratada. En los casos en que no hay patología subyacente se encuentra en una mujer en su vida posterior reproductiva, el tratamiento con antiinflamatorios no esteroideos como para dysmen-orrhea primaria.

En las mujeres adultas, la anticoncepción a largo plazo con las progestinas como inyectables o implantes de depósito conduce a la amenorrea en el 50\% de las mujeres después del primer año de uso que también reduce el número de menstruaciones dolorosas. Una alternativa que se vuelve cada vez más disponibles en entornos de escasos recursos, así es el dispositivo intrauterino de levonorgestrel llamado Mirena®. Más del $80 \%$ de las mujeres con amenorrea será después de un año y antes de la prevalencia de dismenorrea es reducida. Los anticonceptivos a largo plazo basados en progestina se pueden utilizar en dismenorrea primaria. 


\section{La dismenorrea como principal causante de periodos menstruales doloroso}

Vol. 3, núm. 3., (2019)

Paola Katherine Muñoz Cedeño; Jorge Efraín Camacho Desiderio; Ornella Lorena Alvarado Chiquito; Miguel Melquiades Macías Mora

No se debe olvidar que la dismenorrea puede tener causas psicosomáticas, así y evaluar al paciente para este o hacer referencia a un proveedor competente en las instalaciones médicas.

\section{Conclusiones.}

El tratamiento del paciente con dismenorrea incluye una cuidadosa historia de la naturaleza y severidad del dolor, historia menstrual, historia familiar de endometriosis y dismenorrea, historia sexual con antecedentes de uso de anticonceptivos, incluyendo la dispositivo intrauterino. Lo mejor es llevar la historia desde el adolescente tanto con o sin el padre para asegurar la confidencialidad. El doctor debe explicar la anatomía de la pelvis y la necesidad de realizar pruebas para descartar anormalidad. La mayoría de los adolescentes responden bien si la explicación y el examen se realizan en una manera segura y sin prisas. Además, el uso de un pequeño espéculo virginal ayuda a aliviar la ansiedad. Si no se detecta ninguna anomalía en la pelvis y el recto, un antiprostaglandina o un anticonceptivo oral en un adolescente sexualmente activo se prescriben.

El paciente es visto nuevamente en alrededor de tres meses. Si el dolor continúa a pesar de la terapia, o si los hallazgos en el examen pélvico son anormales, grabaciones de ultrasonido, cultivos de genitales para patógenos, y tasa de sedimentación de eritrocitos debe ser obtenida. El diagnostico final generalmente se realiza mediante examen bajo anestesia; histerosalpingografía y diagnóstico laparoscópico se puede realizar Una consulta psicológica es rara vez necesaria y solo se sugeriría después de un examen diagnóstico completo sin revelar patología alguna. Biorretroalimentación, técnicas de relajación y autohipnosis ayudan a estos pacientes. 


\section{La dismenorrea como principal causante de periodos menstruales doloroso}

Vol. 3, núm. 3., (2019)

Paola Katherine Muñoz Cedeño; Jorge Efraín Camacho Desiderio; Ornella Lorena Alvarado Chiquito; Miguel Melquiades Macías Mora

El enfoque general de la dismenorrea debe incluir diagnóstico adecuado para distinguir la dismenorrea primaria de la secundaria, El mito de que "una mujer debe sufrir" debe ser expurgado, y el hecho de que un adolescente no debe ser impedido por la dismenorrea debe ser publicitado

\section{Bibliografía.}

Banikarim, C., Chacko, M., \& Kelder, S. (2000). Prevalence and impact of dysmenorrhea on Hispanic female adolescents. Arch Pediat Adolesc Med , 1226-1229.

Bolívar, J. (2015). Investigación Documental. México. Pax.

Castro, J. (2016). Técnicas Documentales. México. Limusa.

Davila, A. (2015). Concepto de terminos cientificos. Caracas: Oasis.

French, L. (2005). Dysmenorrhea. . Am Fam Physician., 71.

Leyland, N., Casper, R., Laberge, P., \& Singh, S. (2010). Endometriosis: diagnosis and management. J Obstet Gynaecol Can, 1-32.

Morrow, C., \& Naumburg, E. (2009). Dysmenorrhea. Prim Care., 19-32.

P, L., Latthe, M., \& Say, L. (2006). Systematic review of prevalence of chronic pelvic pain: a neglected repro-ductive health morbidity. BMC Public Health, 177.

Wong, C., Farquhar, C., Roberts, H., \& Proctor, M. (2009). Oral contraceptive pill for primary dysmenorrhea. Cochrane Database Syst Rev, 21. 